\title{
Stent Placement for Coronary Stenosis in Kawasaki Disease: Case Report
} and Literature Review

\author{
ELIAS A. ILIADIS, M.D., and CLAIRE S. DUVERNOY, M.D. \\ From the University of Michigan Health System and Ann Arbor Veterans Affairs Health System, Ann Arbor, Michigan
}

\begin{abstract}
A 48-year-old male patient diagnosed with Kawasaki disease in childhood presented with recurrent angina after undergoing coronary artery bypass surgery in 1996. Percutaneous transluminal coronary angioplasty and intracoronary stent placement to a lesion in the proximal ramus intermedius were performed successfully. This case illustrates the complementary nature of percutaneous and surgical myocardial revascularization strategies in appropriately selected patients with Kawasaki disease. (J Interven Cardiol 2002;15:29-32)
\end{abstract}

\section{Case Report}

A 48-year-old male with a past medical history of Kawasaki disease diagnosed in childhood was referred for cardiac catheterization for recurrent angina and abnormal myocardial perfusion study. Prior cardiovascular history was significant for two anterior wall myocardial infarctions in 1987, followed by coronary artery bypass grafting (CABG) in 1996. Surgery consisted of left internal mammary artery (IMA) graft to the left anterior descending (LAD) artery, saphenous vein graft to the first diagonal artery, saphenous vein graft to the first obtuse marginal artery, and saphenous vein graft to the right posterior descending artery.

In July 1998, the patient suffered an inferior wall myocardial infarction treated with angioplasty and stent placement to the saphenous vein graft supplying the right posterior descending artery. The procedure was uneventful, and he was discharged in stable condition. In February 1999, he developed recurrent angina pectoris, which led to intensification of his medical therapy and performance of gated Sestamibi single photon emission computed tomography

Address for reprints: Claire S. Duvernoy, M.D., University of Michigan Health System, Department of Internal Medicine, Division of Cardiology, Veterans Affairs Medical Center, 2215 Fuller Road, Box 11la, Ann Arbor, MI 48015. Fax: (734) 214-0691; e-mail: duvernoy@umich.edu
(SPECT). Myocardial perfusion images revealed reversible, moderate-sized lateral and large inferior defects consistent with ischemia, as well as a large fixed anterior defect consistent with prior infarction. Gated SPECT analysis revealed an ejection fraction of $37 \%$ with mid-anterior wall dyskinesis and apical hypokinesis. Cardiac catheterization was recommended.

Cardiac catheterization showed severe three vessel native coronary artery disease (CAD) consisting of an occluded proximal right coronary artery (RCA) and first obtuse marginal branch, an $80 \%$ to $90 \%$ proximal ramus intermedius stenosis, and a $50 \%$ to $60 \%$ proximal LAD stenosis. Multiple coronary aneurysms were present in the proximal segments (Fig. 1). Of the bypass grafts, the left IMA graft to the LAD artery was $100 \%$ occluded; the saphenous vein graft to the first diagonal artery had a $50 \%$ to $60 \%$ mid-stenosis as well as a $50 \%$ to $60 \%$ anastomotic stenosis. The saphenous vein graft to the right posterior descending artery was patent. Because of his recurrent angina pectoris and abnormal myocardial SPECT, coronary revascularization (redo-CABG versus percutaneous coronary intervention [PCI]) was recommended, and the patient was scheduled for PCI. Percutaneous transluminal coronary angioplasty (PTCA) of the ramus intermedius was performed using an 8Fr EBU guide catheter, a 0.014" ACS Hi-Torque Floppy guidewire (Advanced Cardiovascular Systems Inc., Temecula, CA, USA), and a $2.5 \times 15 \mathrm{~mm}$ SCIMED Coyote balloon (Scimed 


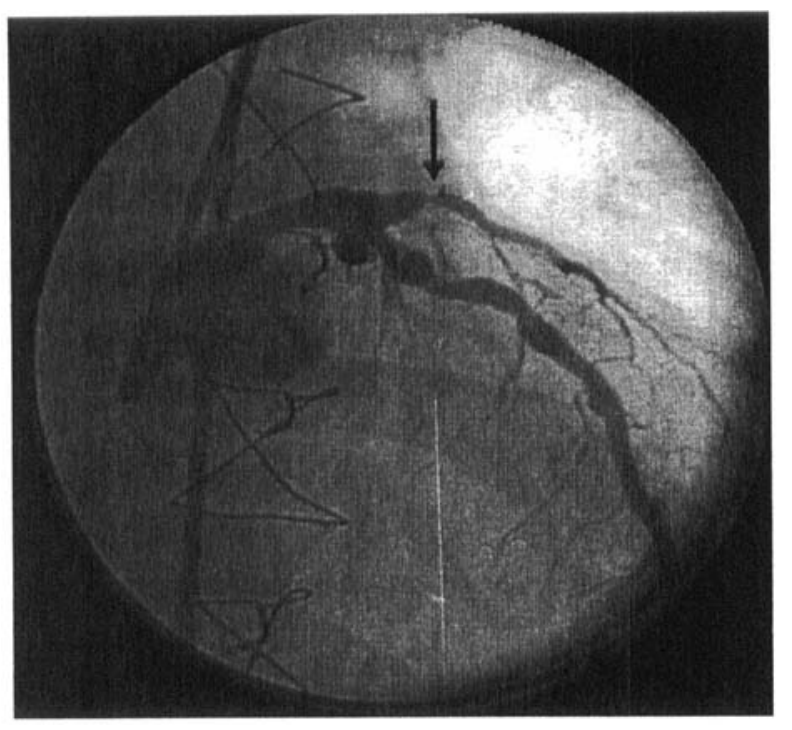

Figure 1. Right anterior oblique (RAO) cranial projection of the left coronary artery showing the stenosis in the proximal ramus intermedius (arrow) and multiple aneurysms in the left anterior descending artery.

Life Systems Inc., Maple Grove, MN, USA). Subsequently, the guidewire was exchanged for a 0.014" Medtronic Mustang floppy guidewire (Medtronic Inc., Minneapolis, MN, USA), and a $2.5 \times 8 \mathrm{~mm}$ ACS Multi-link Duet stent was deployed across the lesion and postdilated using a $3.0 \times 10 \mathrm{~mm}$ SCIMED NC

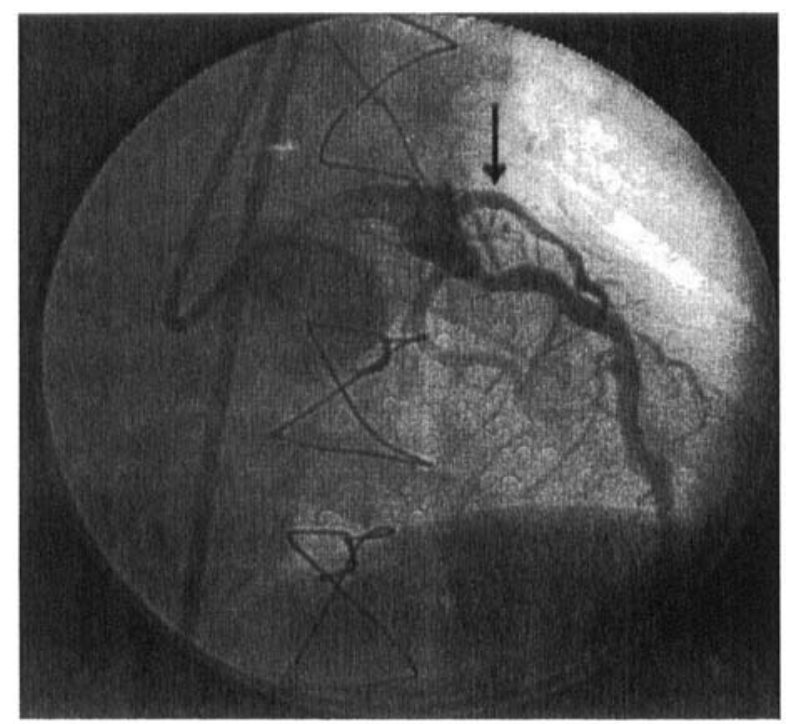

Figure 2. Right anterior oblique (RAO) cranial projection of the left coronary artery after stent deployment and optimization in the proximal ramus intermedius (arrow).
Bandit balloon. Final angiography revealed no residual stenosis, no dissection, and thrombolysis in myocardial infarction (TIMI) III flow (Fig. 2). The patient was discharged to home the following day on clopidogrel $75 \mathrm{mg}$ daily as well as his other medications.

\section{Discussion}

Mucocutaneous lymph node syndrome, or Kawasaki disease, was first described in Japan in 1967 as a generalized vasculitis of unclear etiology. Recently, evidence supports a role for superantigens, which induce extensive $T$ cell proliferation and cytokine production, in the pathogenesis of the disease. ${ }^{1}$ While the acute inflammatory syndrome is generally self-limited, long-term morbidity and mortality is due almost exclusively to the cardiovascular sequelae. ${ }^{2}$ Coronary aneurysms occur in approximately $25 \%$ of untreated patients during the acute phase and are more frequent in young children, males, and those not treated with intravenous immunoglobulin. The majority of these aneurysms do show regression towards normal within a 5-year period; unfortunately, resolution of aneurysms can lead to vessel obstruction or stenosis. ${ }^{3}$ Nonetheless, it is estimated that stenotic lesions will develop in approximately $19 \%$ of all cases with coronary aneurysms and $46 \%$ of those with giant (>8 mm) aneurysms. ${ }^{4}$

Treatment of ischemic coronary heart disease resulting from Kawasaki disease has been controversial. While medical therapy remains the mainstay of treatment for this disease, surgical and percutaneous revascularization strategies have been explored as options for the amelioration of symptoms and to reduce cardiovascular morbidity and mortality. Kitamura et al. reported long-term follow-up data on a group of 168 patients who underwent surgical revascularization for ischemic sequelae of Kawasaki disease, and concluded that patients who received IMA grafts had significantly improved graft patency rates and significantly increased long-term survival rates when compared with patients who received saphenous vein grafts only. ${ }^{5}$ Interestingly, our patient demonstrated early failure of the IMA graft to the LAD coronary artery, possibly because the stenosis demonstrated in his LAD was not critical in nature. Others have reported success with conventional balloon angioplasty for stenoses associated with Kawasaki disease. ${ }^{67}$ Unsuccessful attempts at balloon angioplasty for the treatment of these 


\section{CORONARY STENTING FOR KAWASAKI DISEASE}

lesions also has been reported. ${ }^{8}$ More recently, Sugimura et al. reported success using rotational atherectomy to treat heavily calcified stenoses that had not responded to conventional balloon angioplasty. ${ }^{9}$ However, their finding that coronary aneurysms developed late in two of the four patients treated raises concerns regarding the long-term safety of this treatment modality.

There have been a few recent reports in the literature of successful stent placement for coronary artery stenoses as sequelae of Kawasaki disease. ${ }^{10-12}$ In 1997, Hijazi et al. described the first reported instance of stent implantation in a 16-year-old female with an 80\% LAD stenosis that demonstrated dissection after balloon angioplasty. ${ }^{12}$ One year later, Ishii et al. described the utility of intravascular ultrasound in order to exclude heavy calcification and thus permit successful stent implantation for five out of six children presenting with lesions of the LAD or right coronary arteries. ${ }^{10}$ More recently, Hashmi et al. described successful stent placement into an occluded LAD in a 10year-old child with chest pain and an abnormal myocardial perfusion study. Twelve-month follow-up angiography demonstrated only mild in-stent intimal proliferation. ${ }^{11}$ Finally, Ueno et al. reported on stent implantation for a stenotic as well as aneurysmal coronary artery in a patient with Kawasaki disease. ${ }^{13}$ They deployed a $4.5 \mathrm{~mm}$ stent in the mid-RCA of a patient who had thrombotic occlusion of the vessel and had already sustained three non- $Q$ wave myocardial infarctions to the inferior wall. The treated artery appeared markedly ectatic throughout, whereas the vessel treated in our case contained a discrete aneurysmal segment immediately proximal to the stenotic region, which was of normal caliber. We were especially careful to deploy the stent so that the proximal edge was not hanging out into the aneurysm, but rather just covered the stenotic portion of the vessel. We also decided to treat the patient with aspirin and clopidogrel indefinitely in order to prevent stent thrombosis as well as thrombosis within the aneurysmal segments. Six months following stent placement, the patient remains clinically improved with respect to anginal symptoms and stable at follow-up visits.

\section{Conclusion}

Intracoronary stent placement should be considered as an adjunct or an alternative to other revascularization strategies in the management of patients with ischemic sequelae of Kawasaki disease. Stent placement may offer advantages over conventional balloon angioplasty in these patients.

\section{References}

1. Meissner H, Leung D. Superantigens, conventional antigens, and the etiology of Kawasaki syndrome. Pediatric Infect Dis J 2000;19:91-94.

2. Laupland KB, Dele Davies H. Epidemiology, etiology, and management of Kawasaki disease: State of the art. Pediatric Cardiol 1999;20:177-183.

3. Kato H, Ichinose E, Yoshioka F, et al. Fate of coronary aneurysms in Kawasaki disease: Serial coronary angiography and long-term follow-up study. Am J Cardiol 1982;49: 1758-1766.

4. Kato H, Sugimura T, Akagi $T$, et al. Long-term consequences of Kawasaki disease. A 10- to 21-year follow-up study of 594 patients. Circulation 1996;94:1379-1385.

5. Kitamura S, Kameda Y, Seki T, et al. Long-term outcome of myocardial revascularization in patients with Kawasaki coronary artery disease. A multicenter cooperative study. J Thorac Cardiovasc Surg 1994;107:663-673; discussion 73-74.

6. Min JH, Huh J, Kim YW, et al. Percutaneous transluminal coronary angioplasty in a child with Kawasaki disease. J Korean Med Sci 1998;13:693-695.

7. Ino $T$, Nishimoto $K$, Akimoto $K$, et al. Percutaneous transluminal coronary angioplasty for Kawasaki disease: A case report and literature review. Pediatric Cardiol 1991;12:33-35.

8. Nishimura H, Sawada T, Azuma A, et al. Percutaneous transluminal coronary angioplasty in a patient with Kawasaki disease. A case report of an unsuccessful angioplasty. Jpn Heart J 1992;33:869-873.

9. Sugimura T, Yokoi $\mathrm{H}$, Sato $\mathrm{N}$, et al. Interventional treatment for children with severe coronary artery stenosis with calcification after long-term Kawasaki disease. Circulation 1997;96: 3928-3933.

10. Ishii $\mathbf{M}$, Ueno $\mathbf{T}$, Iemura $\mathbf{M}$, et al. Intracoronary stent implantation with ultrasound guidance for severe coronary artery stenosis after Kawasaki disease. (Abstract) J Am Coll Cardiol 1998;31:326A.

11. Hashmi A, Lazzam C, McCrindle BW, et al. Stenting of coronary artery stenosis in Kawasaki disease. Cathet Cardiovasc Interven 1999;46:333-336.

12. Hijazi Z, Smith J, Fulton J. Stent implantation for coronary artery stenosis after Kawasaki disease. J Invas Cardiol 1997;9:534-536.

13. Ueno $\mathrm{T}$, Kai $\mathrm{H}$, Ikeda $\mathrm{H}$, et al. Coronary stent deployment in a young adult with Kawasaki disease and recurrent myocardial infarction. Clin Cardiol 1999;22:147-149. 